EGU2020-2972

https://doi.org/10.5194/egusphere-egu2020-2972

EGU General Assembly 2020

(c) Author(s) 2020. This work is distributed under

the Creative Commons Attribution 4.0 License.

\title{
Particulate Monitoring and Evaluation of the Low-cost Sensors Performance at the Middle East
}

\author{
Yasser Alshehri ${ }^{1,2}$, Mansour Alghamdi ${ }^{2}$, Mamdouh Khoder ${ }^{2}$, Fahd Almehmadi ${ }^{2}$, Amer Bamuneef ${ }^{3}$, \\ Rayan Moathen ${ }^{3}$, and Georgiy Stenchikov ${ }^{1}$ \\ ${ }^{1}$ King Abdullah University of Science and Technology (KAUST), Saudi Arabia (yasser.alshehri@kaust.edu.sa) \\ ${ }^{2}$ King Abdulaziz University (KAU), Jeddah, Saudi Arabia \\ ${ }^{3}$ The Saudi Authority for Industrial Cities and Technology Zones (MODON), Riyadh, Saudi Arabia
}

\section{Key Words:}

Air Quality; Air Pollution Monitoring; Low-Cost Sensors; Reference Methods, Microsensors, Experimental Campaign

\begin{abstract}
:
Air quality in the Middle East (ME) is strongly affected by desert dust besides anthropogenic pollutants. The health hazards associated with particulate matter (PM) are the most severe in this desert region. The enhancement of Air quality monitoring is needed to implement abatement strategies and stimulate environmental awareness among citizens. Several techniques are used to monitor PM concentration. The air quality monitoring stations (AQMS) equipped with certified instrumentation is the most reliable option. However, AQMSs are quite expensive and require regular maintenance. Another option is low-cost sensors (LCS) that seen as innovative tools for future smart cities. They are cost-effective and allow to increase the spatial coverage of air-quality measurements as the number of conventional AQMS is generally quite small, so the current density of the monitoring stations in the Middle East is low.

In this work, we evaluated the PM air-quality climatology in the major cities in Saudi Arabia (Jeddah, Riyadh, and Dammam) for four years between 2016 and continued until 2020. We used the measurement data that were conducted by the Saudi Authority for Industrial Cities and Technology Zones (MODON) using certified reference AQMS installed inside the suburban areas of the three major cities in Saudi Arabia. Also, we tested the performance of the five LCS systems for eight months, starting in May 2019 and continued until January 2020. For this purpose, we set AQMS with the PM reference instrumentation (based on beta-ray absorption) side-by-side with five different LCS systems (based on light scattering) in the industrial part of Jeddah city. We collected, filtered, validated PM data, and applied standard measurement and calibration procedures.
\end{abstract}

The AQMS measurements show that in Summer, the daily mean PM concentrations exceed the World Health Organization (WHO) limits for PM2.5 and PM10 almost every day in Jeddah, Riyadh, 
and Dammam. The WHO limits are also frequently violated in the winter months. The AQMS measurements reliably show dust storm spikes when PM pollution is extremely high while all the LCSs fail to capture these severe events. We found that LCS and AQMS PM measurements are poorly correlated in Summer, but show slightly better results in fair-weather Winter days when humidity and temperature are low. But they still cannot capture severe dust events. 